[Article]

\title{
活性炭基 $\mathrm{Li}_{2} \mathrm{SO}_{4}$ 水系电解液超级电容器
}

\author{
孙现众张熊张大成 马衍伟* \\ (中国科学院电工研究所, 北京 100190)
}

\begin{abstract}
摘要: 采用中性 $\mathrm{Li}_{2} \mathrm{SO}_{4}$ 水溶液代替 $\mathrm{H}_{2} \mathrm{SO}_{4}$ 和 $\mathrm{KOH}$ 作为电解液制备了活性炭( $\mathrm{AC}$ )基对称型超级电容器, 使水系 超级电容器的工作电压由 $1.0 \mathrm{~V}$ 提高到了 $1.6 \mathrm{~V}$. 采用循环伏安和充放电测试研究了电容器的稳定电化学窗口. 电化学充放电测试表明电容器在 $0.25 \mathrm{~A} \cdot \mathrm{g}^{-1}$ 电流密度下单电极比容量可达 $129 \mathrm{~F} \cdot \mathrm{g}^{-1}$, 在功率密度为 $160 \mathrm{~W}$. $\mathrm{kg}^{-1}$ 时能量密度达到 $10 \mathrm{Wh} \cdot \mathrm{kg}^{-1}$ (以正负极活性物质的总质量计). $1.6 \mathrm{~V}$ 恒压充电 $1 \mathrm{~h}$ 后电容器漏电流为 0.22 $\mathrm{mA}$. 超级电容器的库仑效率接近 $100 \%$, 充放电循环 5000 次后容量仍可保持在 $92 \%$ 以上. 研究了电解液的浓 度对电容器电化学性能的影响, 发现随着 $\mathrm{Li}_{2} \mathrm{SO}_{4}$ 浓度的增大电容器的电荷转移电阻显著减小, 大电流充放电性 能提高. 活性炭基 $\mathrm{Li}_{2} \mathrm{SO}_{4}$ 水系电解液超级电容器具有工作电压高、能量密度高和对环境友好等优点, 因此有很 好的产业化前景.
\end{abstract}

关键词: 超级电容器; 活性炭; 中性水系电解液; $\mathrm{Li}_{2} \mathrm{SO}_{4}$

中图分类号: $\mathrm{O} 646$

\section{Activated Carbon-Based Supercapacitors Using $\mathrm{Li}_{2} \mathrm{SO}_{4}$ Aqueous Electrolyte}

\author{
SUN Xian-Zhong ZHANG Xiong ZHANG Da-Cheng MA Yan-Wei* \\ (Institute of Electrical Engineering, Chinese Academy of Sciences, Beijing 100190, P. R. China)
}

\begin{abstract}
In this work, we prepared activated carbon-based symmetric supercapacitors using $\mathrm{Li}_{2} \mathrm{SO}_{4}$ aqueous electrolyte instead of $\mathrm{H}_{2} \mathrm{SO}_{4}$ and $\mathrm{KOH}$, and obtained devices with an improved working voltage of $1.6 \mathrm{~V}$ from $1.0 \mathrm{~V}$. Cyclic voltammetry and galvanostatic charging/discharging measurements were used to study the electrochemical properties. The results showed that the electrode specific capacitance can reach $129 \mathrm{~F} \cdot \mathrm{g}^{-1}$, and the energy density can be as high as $10 \mathrm{Wh} \cdot \mathrm{kg}^{-1}$ at a power density of $160 \mathrm{Wh} \cdot \mathrm{kg}^{-1}$. Electrochemical impedance analysis measurements showed that the charge-transfer resistance of the capacitors decreased markedly with the increase of the concentration of $\mathrm{Li}_{2} \mathrm{SO}_{4}$, and the rate capability improved accordingly. The leakage current of the supercapacitor was $0.22 \mathrm{~mA}$ after constant-voltage charging at $1.6 \mathrm{~V}$ for $1 \mathrm{~h}$, and the columbic efficiency was nearly $100 \%$. The capacitance of the supercapacitor remained above $90 \%$ after 5000 charge-discharge cycles. Activated carbon-based supercapacitors using $\mathrm{Li}_{2} \mathrm{SO}_{4}$ aqueous electrolyte have many advantages, such as high working voltage, high energy density, and environmental compatibility, and therefore have good industrialization prospects.
\end{abstract}

Key Words: Supercapacitor; Activated carbon; Neutral aqueous electrolyte; $\mathrm{Li}_{2} \mathrm{SO}_{4}$

\footnotetext{
Received: October 11, 2011; Revised: December 7, 2011; Published on Web: December 13, 2011.

"Corresponding author. Email: ywma@mail.iee.ac.cn; Tel: +86-10-82547129; Fax: +86-10-82547137.

The project was supported by the Knowledge Innovation Program of the Chinese Academy of Sciences (KJCX2-YW-W26), Science and Technology Project of Beijing, China (Z111100056011007), and National Natural Science Foundation of China (21001103, 51025726). 中国科学院知识创新工程重要方向项目(KJCX2-YW-W26), 北京市科技计划项目(Z111100056011007)和国家自然科学基金(21001103, 51025726)资助
}

(C) Editorial office of Acta Physico-Chimica Sinica 


\section{1 引 言}

超级电容器也称电化学电容器, 是一种新型的 储能元件, 兼有常规电容器的功率密度大和化学电 源能量密度高的优点, 可快速充放电, 使用寿命长, 既可以单独使用作为主要的储能器件, 也可以与充 电电池组成复合电源系统, 在新能源发电、电动汽 车、信息技术、航空航天和国防等领域都有广阔的 应用前景. ${ }^{1-4}$

超级电容器根据电解液的不同可分为有机体 系和水溶液体系超级电容器 3,5 前者电化学窗口较 大(工作电压 2-4 V), 但电解液价格比较昂贵, 制作 电容器时需要干燥的生产环境, 工艺成本比较高; 而水系电解液成本较低, 电容器的生产工艺简单, 但电解液电化学窗口较小, 工作电压一般为 $1.0 \mathrm{~V}$. 根据公式 $E=1 / 2 C U^{2}$ 可知, 超级电容器的能量密度 $E$ 与电容 $C$ 成正比, 与电压 $U$ 的平方成正比, 因此要提 高超级电容器的能量密度, 可以从提高电极材料的 比容量和超级电容器的工作电压两个方面入手. 前 者可以通过高比容量电极材料的制备和改性来提 高材料的比容量和能量密度, ${ }^{6-13}$ 后者则可通过采用水 系非对称结构(如活性炭 $(\mathrm{AC}) / \mathrm{MnO}_{2}{ }^{14,15} \mathrm{AC} / \mathrm{MoO}_{3},{ }^{16,17}$ $\mathrm{AC} / \mathrm{Ni}(\mathrm{OH})_{2}{ }^{18} \mathrm{AC} / \mathrm{LiMn}_{2} \mathrm{O}_{4},{ }^{19} \mathrm{WO}_{3} / \mathrm{VO}_{x},{ }^{20} \mathrm{MnFe}_{2} \mathrm{O}_{4} /$ $\mathrm{LiMn}_{2} \mathrm{O}_{4}{ }^{21}$ 等)或者有机体系来提高电容器的电压窗 口.

活性炭基超级电容器的水系电解液通常采用 $\mathrm{H}_{2} \mathrm{SO}_{4}$ 和 $\mathrm{KOH}$ 水溶液, 而中性电解液如碱金属硫酸 盐的水溶液则是用于非对称超级电容器中. ${ }^{22,23} \mathrm{Qu}$ 等 ${ }^{24}$ 采用三电极体系研究了活性炭电极在中性的 $\mathrm{Li}_{2} \mathrm{SO}_{4} 、 \mathrm{Na}_{2} \mathrm{SO}_{4} 、 \mathrm{~K}_{2} \mathrm{SO}_{4}$ 水溶液中的电化学性能, 发现 在三种电解液中电极的容量和循环性能差别不 大, 而 $\mathrm{K}_{2} \mathrm{SO}_{4}$ 水溶液中电极的大电流放电性能较好. Demarconnay 等 ${ }^{25}$ 从 $\mathrm{AC} / \mathrm{MnO}_{2}$ 非对称型超级电容器 的工作中得到启发, 首次将 $\mathrm{Na}_{2} \mathrm{SO}_{4}$ 电解液应用于制 备活性炭对称型超级电容器, 由于电极在中性电解 液中的析氧/析氢过电位与在 $\mathrm{H}_{2} \mathrm{SO}_{4}$ 和 $\mathrm{KOH}$ 水溶液 中不同, 从而可将水系超级电容器的工作电压由 $1.0 \mathrm{~V}$ 提高至 $1.6 \mathrm{~V}$. 由于 $\mathrm{Na}_{2} \mathrm{SO}_{4}$ 在水中的溶解度较 低, 且随温度变化比较大 $\left(1.4 \mathrm{~mol} \cdot \mathrm{L}^{-1}, 20^{\circ} \mathrm{C} ; 0.35\right.$ $\left.\mathrm{mol} \cdot \mathrm{L}^{-1}, 0{ }^{\circ} \mathrm{C}\right)$, 限制了其在低温环境中的应用. 为 此, 本文选择了溶解度更高且低温溶解度稳定的 $\mathrm{Li}_{2} \mathrm{SO}_{4}\left(3.2 \mathrm{~mol} \cdot \mathrm{L}^{-1}, 20^{\circ} \mathrm{C} ; 3.3 \mathrm{~mol} \cdot \mathrm{L}^{-1}, 0{ }^{\circ} \mathrm{C}\right)$ 作为超 级电容器的电解质盐, 制备了电容器的器件, 并研 究了其电化学性能.

\section{2 实 验}

电容器的正负极活性物质均采用上海合达炭 素材料有限公司生产的比表面积为 $2500 \mathrm{~m}^{2} \cdot \mathrm{g}^{-1}$ 的 活性炭, 导电剂为上海荣仲实业有限公司提供的 $50 \%$ 压缩乙炔黑, 粘结剂选用 Aldrich 公司提供的浓 度为 $60 \%$ 的聚四氟乙烯乳液, 隔膜为深圳市比源电 子有限公司提供的超级电容器用聚丙烯隔膜, 电解 液为采用广东光华化学厂生产的分析纯一水合硫 酸锂配制成的 $0.5-2.0 \mathrm{~mol} \cdot \mathrm{L}^{-1}$ 的 $\mathrm{Li}_{2} \mathrm{SO}_{4}$ 水溶液(若 无特别说明, 浓度均为 $\left.1.0 \mathrm{~mol} \cdot \mathrm{L}^{-1}\right)$. 超级电容器的 制备方法为: 先将活性炭、乙炔黑和聚四氟乙烯按 70:20:10 的质量比混合均匀, 加入异丙醇搅拌成糊 状, 加热至 $60^{\circ} \mathrm{C}$ 破乳, 用手动轧机擀成薄膜压覆在 不锈钢网上, 放入真空干燥箱中在 $120{ }^{\circ} \mathrm{C}$ 下干燥 8 $\mathrm{h}$, 冲制成直径为 $14 \mathrm{~mm}$ 的极片. 将极片和隔膜放入 扣式电池壳中, 滴加电解液后封口组装成扣式电容 器.

在上海辰华公司 CHI660c 电化学工作站上对电 容器进行了电化学阻抗、循环伏安等测试. 电化学 阻抗测试采用的交流信号振幅为 $5 \mathrm{mV}$, 频率范围为 0.01-100 kHz; 循环伏安测试采用的扫描速率为 5 $\mathrm{mV} \cdot \mathrm{s}^{-1}$, 扫描的起始电压为 $0 \mathrm{~V}$, 截止电压为 1.0-2.0 V. 电容器的恒流充放电、循环性能和漏电 流测试均采用了美国 Arbin 公司 MSTAT 4 电化学测 试系统. 漏电流的测试方法为先用恒流方法将电容 器充电至 $1.6 \mathrm{~V}$, 然后用恒压充电的方法充电 $1 \mathrm{~h}$, 恒 压充电过程中的电流即为漏电流. 采用两电极系统 和三电极系统测试了在充电过程中电位变化的过 程以及活性炭电极的循环伏安曲线, 参比电极为饱 和甘录电极 (SCE) 并转化为相对于标准氢电极的电 位( vs NHE), 三电极系统的对电极为铂片.

\section{3 结果与讨论}

图 1 所示为不同截止电压下电容器的循环伏安 曲线, 可以看到截止电压在 1.0-1.6 V 范围内循环伏 安曲线都为近似对称的矩形曲线, 表明该电容器具 备良好的电容特性. 扫描过程中未出现明显的氧化还原峰, 这说明电容器电荷的存储是双电层机制. 随着电容器的充电截止电压增大 $2.0 \mathrm{~V}$, 正向电流显 著增加, 在反向扫描过程中也出现了相应的电流增 大部分, 这表明电容器的正极和/或负极发生了氧化 还原反应.

图 2 所示为相应的不同截止电压下的恒流充放 
电曲线, 电流密度为 $0.25 \mathrm{~A} \cdot \mathrm{g}^{-1}$ (以正负极活性物质 的总质量计). 可以看到, 在较小的电压窗口下充放 电曲线表现为良好的线性特性和对称性等双电层 电容的特征; 随着充电电压大于 $1.8 \mathrm{~V}$, 充电曲线斜 率逐渐增加而偏离了线性特性, 这表明电极表面在 发生双电层电荷存储的同时还发生了部分的电化 学氧化还原反应.

为了研究超级电容器的充放电过程, 我们利用 两电极系统在对电容器充电的同时还监测了正负 极电位的变化, 如图 3 所示. 在充电过程中电容器电 压从 $0 \mathrm{~V}$ 增大到 $2.0 \mathrm{~V}$, 相应的, 相对于 $\mathrm{NHE}$ 参比电 极, 正极电位从 $+0.29 \mathrm{~V}$ 升高到 $+1.14 \mathrm{~V}$, 同时负极电 位从 $+0.29 \mathrm{~V}$ 降低到 $-0.86 \mathrm{~V}$, 据此, 我们利用三电极 系统测试了活性炭电极在 $-0.86-+1.14 \mathrm{~V}$ ( vs NHE) 范围内的循环伏安曲线 (图 4). 从图 3 中发现, 在 $0-1.7 \mathrm{~V}$ 的电压范围内电极的电位与电容器的电压 成线性关系, 但是电压超过 $1.7 \mathrm{~V}$ 后正极电位开始出 现电位平台; 由循环伏安曲线可以看到, 正向扫描

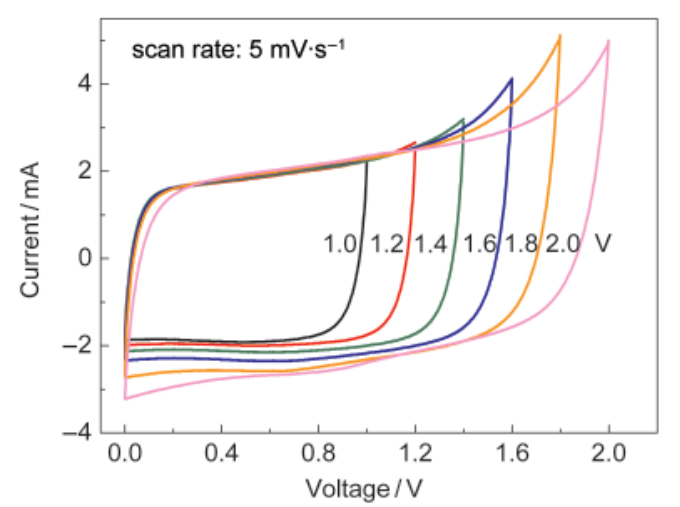

图 1 1.0-2.0 V 截止电压下电容器的循环伏安曲线

Fig.1 Cyclic voltammograms of capacitor with cut-off voltages from 1.0 to $2.0 \mathrm{~V}$

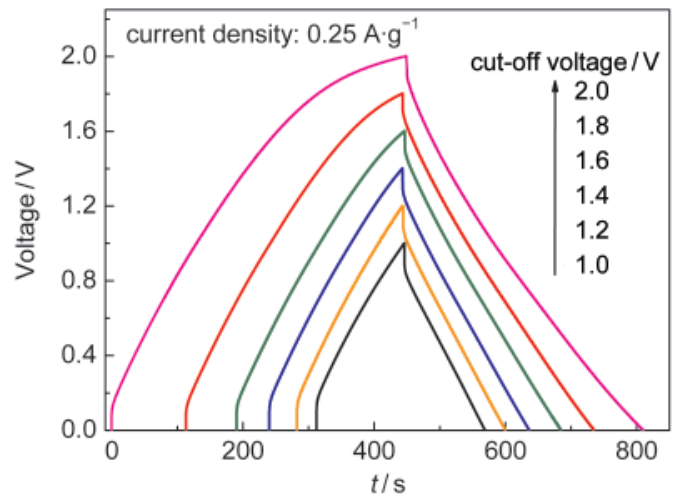

图 $21.0-2.0 \mathrm{~V}$ 截止电压下电容器的恒流充放电曲线

Fig.2 Galvanostatic charge/discharge curves of capacitor varied with cut-off voltages from 1.0 to $2.0 \mathrm{~V}$
过程中当电极电位高于 $+1.1 \mathrm{~V}$ (对应于图 3 所示 1.7 $\mathrm{V}$ 充电电压下的正极电位) 时响应电流显著增大, 这 表明在正极发生了活性炭电极的氧化反应. 在反向 扫描过程中, 当电极电位低于 $-0.65 \mathrm{~V}$ (对应于 $1.7 \mathrm{~V}$ 充电电压下的负极电位) 时, 响应电流迅速增大, 表 明发生了水的分解和在活性炭中氢的化学吸附, ${ }^{25,26}$ 这一过程可以表示为: $\mathrm{H}_{2} \mathrm{O}+\mathrm{e}^{-} \rightarrow \mathrm{H}+\mathrm{OH}^{-}$和 $\mathrm{C}+\mathrm{H} \rightleftharpoons$ $\mathrm{CH}_{\mathrm{ad}}$, 其中 $\mathrm{H}_{\mathrm{ad}}$ 为活性炭的吸附氢. 为此, 为了提高电 容器的库仑效率, 避免电极发生的不可逆氧化反应 降低电容器的循环性能, 我们选定 $\mathrm{Li}_{2} \mathrm{SO}_{4}$ 体系电容 器的稳定电化学窗口为 $0-1.6 \mathrm{~V}$.

图 5 所示为电容器的特征的最初三个循环的充 放电曲线 $\left(0.25 \mathrm{~A} \cdot \mathrm{g}^{-1}\right)$. 在第一个充放电循环, 电荷效 率较低, 这可以归结为活性炭材料表面含氧基团发 生了不可逆的氧化反应. ${ }^{27}$ 经首次电化学活化后, 电 容器充放电过程的电荷效率大大提高, 充放电曲线 具有良好的三角形对称性分布, 电容器电压随充放 电时间的变化呈线性关系.

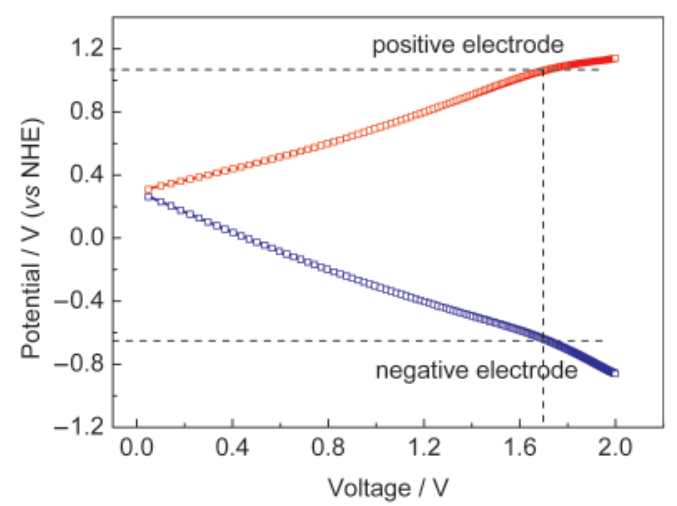

图 3 充电过程中正、负极电位相对于电容器电压的变化

Fig.3 Potential plots of the positive and negative electrodes vs voltage during charging process

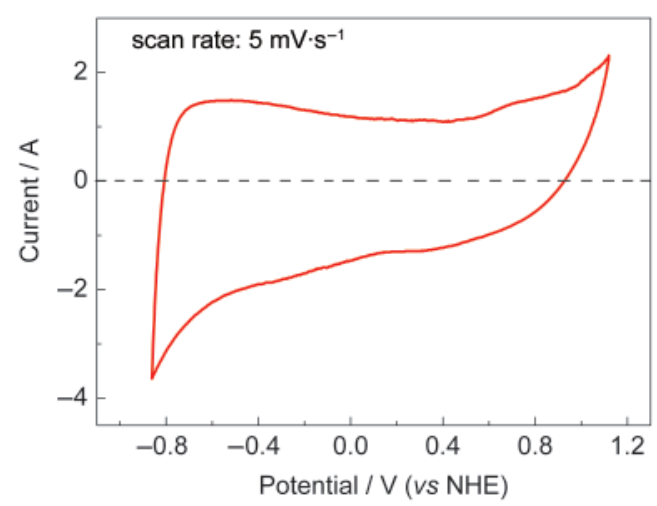

图 4 三电极体系活性炭电极在 $\mathrm{Li}_{2} \mathrm{SO}_{4}$ 电解液中的 循环伏安曲线

Fig.4 Three-electrode cyclic voltammogramms of activated carbon electrode in $\mathrm{Li}_{2} \mathrm{SO}_{4}$ electrolyte 


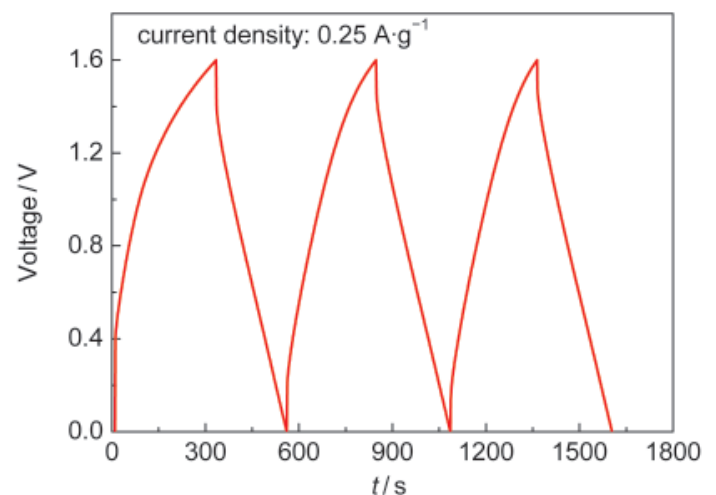

图 5 电容器的恒流充放电曲线

Fig.5 Galvanostatic charge/discharge curves of capacitor

图 6 所示为不同浓度 $\mathrm{Li}_{2} \mathrm{SO}_{4}$ 电解液的电容器电 化学阻抗谱图. 电容器的阻抗谱由高频区的半圆 弧、中低频区中倾角约为 $45^{\circ}$ 的斜线和低频区与虚 轴接近平行的直线等三部分组成. 通常认为半圆弧 主要对应于电解液和电极的本征阻抗和电荷转移 阻抗, 半圆弧在实轴的截距为电容器的内阻 $R_{\mathrm{s}}$, 其值 包括溶液电阻、活性炭材料的电阻和电极片与集流 体之间以及活性炭颗粒与导电剂之间的接触电阻 等; 半圆弧的直径为电荷转移电阻 $R_{\mathrm{ct}}$, 代表在电极/ 电解液界面电荷转移的动力学电阻或电解液和多 孔电极的本征电荷转移电阻. 中低频区的斜线对应 于离子在固体颗粒内有限长度扩散的 Warburg 阻抗 $Z_{\mathrm{w}}$. 低频区的直线部分代表了器件的电容特性, 斜率 越大表示越接近于理想电容. ${ }^{28}$ 从图中可以看出, 超 级电容器的谱图在低频段趋近于与实轴垂直, 对应 于 $0.5-2.0 \mathrm{~mol} \cdot \mathrm{L}^{-1}$ 的 $\mathrm{Li}_{2} \mathrm{SO}_{4}$ 电解液谱图低频段与实 轴的夹角分别为 $84.5^{\circ} 、 85.8^{\circ} 、 86.0^{\circ}$ 和 $86.6^{\circ}$, 表明活 性炭电极趋于完全极化状态, 显示了良好的电容特

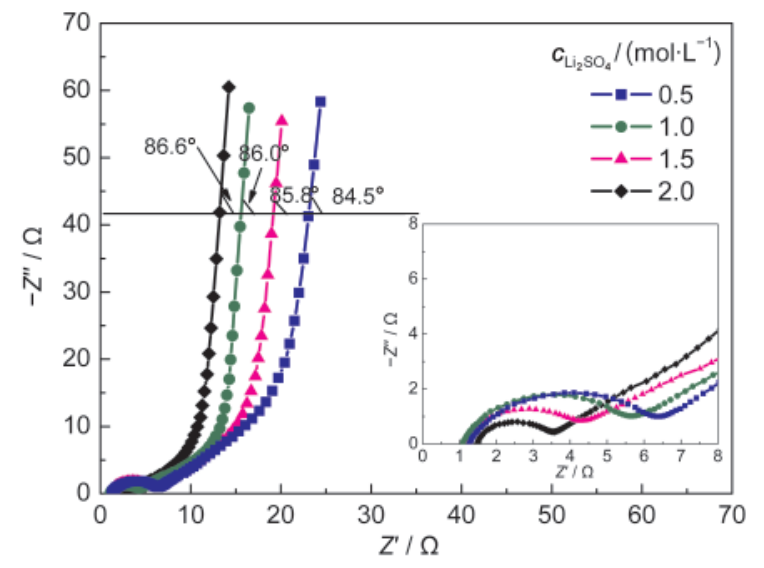

图 6 电容器的电化学阻抗谱图和高频区的放大图(插图)

Fig.6 Nyquist plots of capacitors and enlarged high-frequency parts (inset)

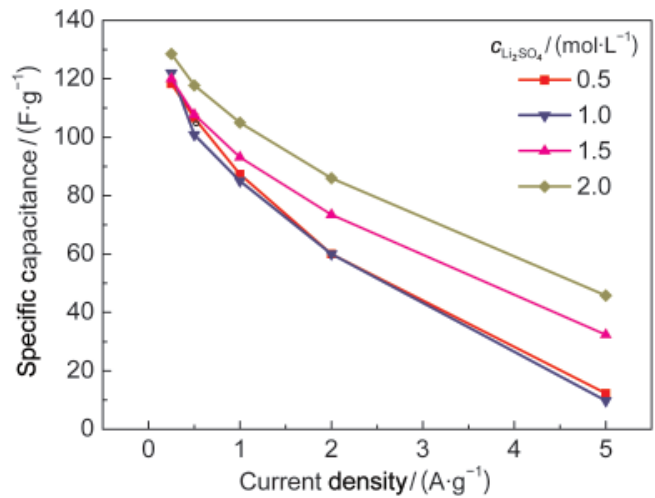

图 7 采用不同浓度 $\mathrm{Li}_{2} \mathrm{SO}_{4}$ 电解液的活性炭电极的 比容量与放电电流密度关系曲线

Fig.7 Relationship between the specific capacitance of

AC electrode in $\mathrm{Li}_{2} \mathrm{SO}_{4}$ electrolyte with different concentrations and discharging current density

性, 并且随电解液浓度的增加电容特征更为显著, 同时 Warburg 阻抗减小. 图 6 插图显示, 随着电解液 浓度的增加, 阻抗谱半圆弧的直径显著减小, 表明 随着电解质离子浓度的增加, 电极的电荷转移电阻 $R_{\mathrm{ct}}$ 减小.

根据公式 $C=4 I t /(M \Delta U)$ 可以计算出电容器电极 的比容量, 其中, $I$ 为放电电流, $t$ 为放电时间, $\Delta U$ 为 充放电的电压窗口, $M$ 为正、负极活性物质的总质 量. ${ }^{25}$ 在 $0.25 \mathrm{~A} \cdot \mathrm{g}^{-1}$ 电流下电极比容量可达 $129 \mathrm{~F} \cdot \mathrm{g}^{-1}$, 在低电流下电极比容量比较接近, 但由于阻抗的差 异, 随着放电电流的增加, 低浓度电解液的电容器 比容量下降较快, 而采用高浓度电解液的电容器 的比电容仍能保持较高的值 (见图 7). 根据公式 $P=\Delta U I / 2 M$ 和 $E=P t$ 可以计算出电容器的功率密度 $P$ 和能量密度 $E,{ }^{28}$ 由图 8 所示的电容器的 Ragone 图可 以看出, 电容器的能量密度可达 $10 \mathrm{Wh} \cdot \mathrm{kg}^{-1}$, 随着放

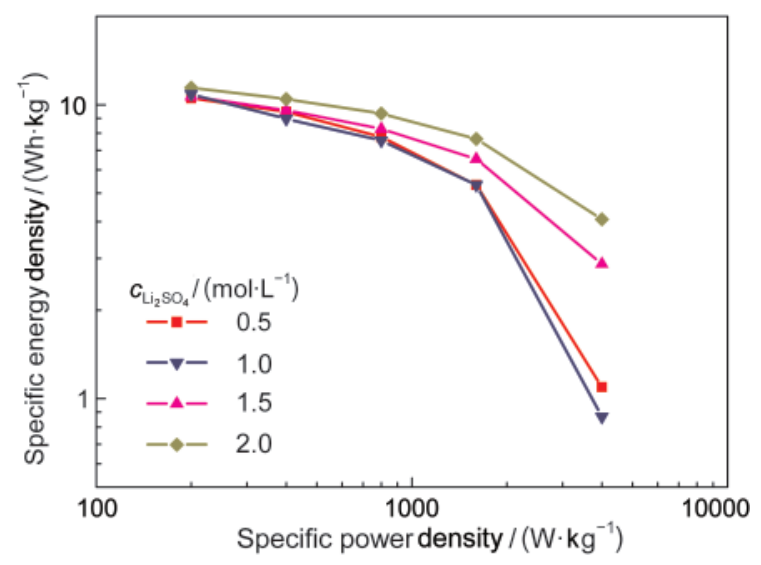

图 8 不同浓度 $\mathrm{Li}_{2} \mathrm{SO}_{4}$ 电解液电容器的 Ragone 曲线 Fig.8 Ragone plots of capacitors with different $\mathrm{Li}_{2} \mathrm{SO}_{4}$ electrolyte concentrations 
电倍率的增大, 电容器放电比容量均有不同程度的 降低, 但总体而言, 采用高浓度电解液的电容器由 于阻抗较低其大电流性能更佳.

电容器的漏电主要有以下三方面的原因: (1) 由 于极片或电解液中存在杂质而在电极表面形成微 电化学电池; (2) 由电势差和电解质离子浓度梯度引 起的双电层电荷向溶液本体扩散, ${ }^{29}$ (3) 集流体和壳 体由于毛刺等刺穿隔膜引起微短路. 采用 $\mathrm{Li}_{2} \mathrm{SO}_{4}$ 电 解液制备的电容器漏电流很小, 如图 9 所示, 电容器 的漏电流在 $1.6 \mathrm{~V}$ 恒压充电条件下 $2 \mathrm{~min}$ 内由 2.2 $\mathrm{mA}$ 迅速降低至 $0.5 \mathrm{~mA}$ 以下, 随着恒压时间的增加 漏电流逐渐减小, $10 \mathrm{~min}$ 后基本趋于稳定, $1 \mathrm{~h}$ 之后 漏电流降低至 $0.22 \mathrm{~mA}$.

图 10 为电容器的充放电循环性能曲线, 电流密 度为 $1 \mathrm{~A} \cdot \mathrm{g}^{-1}$. 电容 100 次循环后容量保持率降低 $96.6 \%, 5000$ 次循环后容量保持率缓慢降低至 $92.2 \%$, 比 100 次循环时仅衰减了 $4.4 \%$; 库仑效率 100 次循环后由 $97.0 \%$ 上升到 $99.3 \%$, 此后基本保持

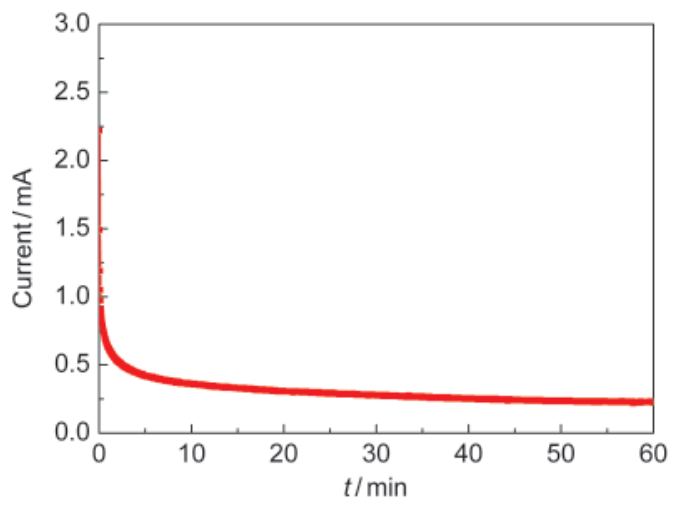

图9电容器的漏电流曲线

Fig.9 Leakage current curve of capacitor

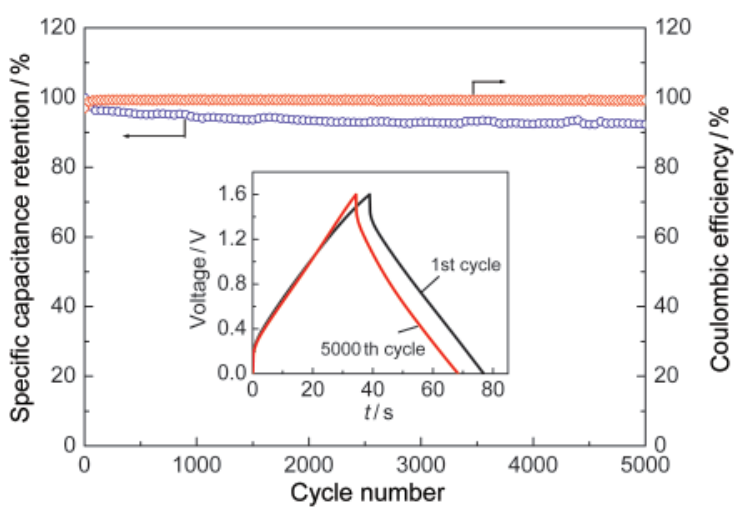

图 10 电容器比电容保持率和库仑效率与循环次数的关系

Fig.10 Evolution of the specific capacitance retention and coulombic efficiency versus the number of cycles Inset is the charge/discharge curves at current density of $1 \mathrm{~A} \cdot \mathrm{g}^{-1}$.
不变, 这说明电容器具有良好的循环稳定性. 由图 10 插图中还可以看到, 5000 次循环后充放电曲线的 线性特征更加明显, 对称性更好, 而这一变化可归 因于活性炭表面的含氧基团发生的不可逆法拉第 反应, ${ }^{27}$ 在经过多次充放电循环后, 这部分含氧基团 被消耗, 从而表现出了完全的双层电容性质.

\section{4 结 论}

采用中性的 $\mathrm{Li}_{2} \mathrm{SO}_{4}$ 水溶液电解液制备活性炭基 对称型超级电容器. 采用不同截止电压的循环伏安 曲线和充放电曲线表明, 电容器虽然可以在 $0-2.0$ $\mathrm{V}$ 范围内充放电, 但电压超过 $1.7 \mathrm{~V}$ 后正极的活性炭 会发生显著的氧化反应; 电容器在 $1.6 \mathrm{~V}$ 的电压下 循环充放电 5000 次都保持了较高的容量, 这表明选 择 $1.6 \mathrm{~V}$ 的工作电压是合适的. 电容器在 $0.25 \mathrm{~A} \cdot \mathrm{g}^{-1}$ 电流密度下单电极比容量可达 $129 \mathrm{~F} \cdot \mathrm{g}^{-1}$, 在 $160 \mathrm{~W}$. $\mathrm{kg}^{-1}$ 时电容器能量密度达到 $10 \mathrm{Wh} \cdot \mathrm{kg}^{-1}$ (以正负极活 性物质的总质量计). 电化学阻抗谱和充放电测试表 明, 电容器表现了良好的电容特性, 且电容器的阻 抗随 $\mathrm{Li}_{2} \mathrm{SO}_{4}$ 浓度的增大而减小, 相应地大电流充放 电性能提高.

\section{References}

(1) Miller, J. R.; Simon, P. Science 2008, 321, 651.

(2) Ji, Q. Q.; Guo, P. Z.; Zhao, X. S. Acta Phys. -Chim. Sin. 2010, 26, 1254. [季倩倩, 郭培志, 赵修松. 物理化学学报, 2010, 26, 1254.]

(3) Zhu, Y.; Murali, S.; Stoller, M. D.; Ganesh, K. J.; Cai, W.; Ferreira, P. J.; Pirkle, A.; Wallace, R. M.; Cychosz, K. A.; Thommes, M.; Su, D.; Stach, E. A.; Ruoff, R. S. Science 2011, 332, 1537.

(4) Simon, P.; Gogotsi, Y. Nat. Mater. 2008, 7, 845.

(5) Chen, Y.; Zhang, X.; Yu, P.; Ma, Y. W. J. Power Sources 2010, 195, 3031 .

(6) Chen, Y.; Zhang, X.; Zhang, D. C.; Yu, P.; Ma, Y. W. Carbon 2011, 49, 573.

(7) Zhang, D. C.; Zhang, X.; Chen, Y.; Yu, P.; Wang, C. H.; Ma, Y. W. J. Power Sources 2011, 196, 5990.

(8) Yu, L. Q.; Chen, S. L.; Chang, S.; Li, Y. H.; Gao, Y. Y.; Wang, G. L.; Cao, D. X. Acta Phys. -Chim. Sin. 2011, 27, 615. [于丽 秋, 陈书礼, 常 莎, 李云虎, 高胤义, 王贵领, 曹殿学. 物理化 学学报, 2011, 27, 615.]

(9) Wang, H.; Gao, Q.; Jiang, L. Small 2011, 7, 2454.

(10) Lu, X. H.; Zheng, D. Z.; Zhai, T.; Liu, Z. Q.; Huang, Y. Y.; Xie, S. L.; Tong, Y. X. Energ. Environ. Sci. 2011, 4, 2915.

(11) Xu, B.; Zhang, H.; Cao, G. P.; Zhang, W. F.; Yang, Y. S. Prog. Chem. 2011, 23, 605. [徐斌, 张 浩, 曹高萍, 张文峰, 杨裕 
生. 化学进展, 2011, 23, 605.]

(12) Xu, B.; Yue, S. F.; Sui, Z. Y.; Zhang, X. T.; Hou, S. S.; Cao, G. P.; Yang, Y. S. Energ. Environ. Sci. 2011, 4, 2826.

(13) Lin, P.; She, Q. J.; Hong, B. L.; Liu, X. J.; Shi, Y. N.; Shi, Z.; Zheng, M. S.; Dong, Q. F. J. Electrochem. Soc. 2010, 157, A818.

(14) Deng, L.; Zhu, G.; Wang, J.; Kang, L.; Liu, Z. H.; Yang, Z.; Wang, Z. J. Power Sources doi: 10.1016/j.jpowsour. 2011.09.005.

(15) Li, W. C.; Gao, P. C.; Lu, A. H. J. Power Sources 2011, 196, 4095.

(16) Brezesinski, T.; Wang, J.; Tolbert, S. H.; Dunn, B. Nat. Mater. 2010, 9,146

(17) Tang, W.; Liu, L.; Tian, S.; Li, L.; Yue, Y.; Wu, Y.; Zhu, K. Chem. Commun. 2011, 47, 10058.

(18) Hu, G. X.; Li, C. X.; Gong, H. J. Power Sources 2010, 195 , 6977.

(19) Wen, Z. B.; Tian, S.; Qu, Q. T.; Wu, Y. P. Prog. Chem. 2011, 23, 589. [温祖标, 田 舒, 曲群婷, 吴宇平. 化学进展, 2011, 23, 589.]

(20) Li, J. M.; Chang, K. H.; Hu, C. C. Electrochem. Commun. 2010, 12,1800
(21) Lin, Y. P.; Wu, N. L. J. Power Sources 2011, 196, 851.

(22) Mosqueda, H. A.; Crosnier, O.; Athouël, L.; Dandeville, Y.; Scudeller, Y.; Guillemet, P.; Schleich, D. M.; Brousse, T. Electrochim. Acta 2010, 55, 7479.

(23) Zhang, X.; Yang, W. S.; Ma, Y. W. Electrochem. Solid. St. 2009, 12, A95.

(24) Qu, Q. T.; Wang, B.; Yang, L. C.; Shi, Y.; Tian, S.; Wu, Y. P. Electrochem. Commun. 2008, 10, 1652.

(25) Demarconnay, L.; Raymundo-Piñero, E.; Béguin, F. Electrochem. Commun. 2010, 12, 1275.

(26) Béguin, F.; Jurewicz, K.; Frackowiak, E. Appl. Phys. A 2004, 78, 981.

(27) Khomenko, V.; Raymundo-Piñero, E.; Béguin, F. J. Power Sources 2010, 195, 4234 .

(28) Xu, C.; Du, H.; Li, B.; Kang, F.; Zeng, Y. J. Electrochem. Soc. 2009, 156, A435.

(29) Li, J.; Lai, Y. Q.; Jin, X. D.; Peng, R. F.; Liu, Y. X. Chinese Battery Industry 2010, 15, 131. [李晶, 赖延清, 金旭东, 彭 汝芳, 刘业翔. 电池工业, 2010, 15, 131.] 